\title{
Evidence of an anomalous metallic phase in a high temperature superconducting device
}

\author{
Quantum coherence across bosonic superconductor-anomalous \\ metal-insulator transitions \\ Authors: Chao Yang, Yi Liu, Yang Wang, Liu Feng, Qianmei He, Jian Sun, Yue \\ Tang, Chunchun Wu, Jie Xiong, Wanli Zhang, Xi Lin, Hong Yao, Haiwen Liu, \\ Gustavo Fernandes, Jimmy Xu, James M. Valles Jr., Jian Wang, and Yanrong Li \\ arXiv:1901.07706
}

\section{Recommended with a Commentary by Steven A. Kivelson, Stanford University, Stanford, CA 94205}

Considered as a quantum phase of matter, a metal is defined by finite ohmic dissipation in the limit that the temperature, $T \rightarrow 0$. The one clear theoretical paradigm of a metallic phase is the Drude metal of weakly interacting quasiparticles in weak disorder. On the other hand, there have been, for many years, experimental indications of various forms of anomalous metallic phases, with behaviors that are not remotely consistent with a simple Drude description. In particular, there has recently been considerable interest in an anomalous metallic phase that appears in the neighborhood of an apparent quantum superconductor to metal transition in 2D systems tuned by varying a number of different quantum tuning parameters. [2] Such systems show clear signs that the dominant charge carrying excitations are locally superconducting collective modes, so the major issue is whether their failure to condense into a true superconducting groundstate as $T \rightarrow 0$ is intrinsic or extrinsic. However, there have also been lingering uncertainties concerning the correct interpretation of these observations as they typically involve delicate electronic states and very low temperatures, and thus there have been persistent concerns about non-equilibrium effects and contamination by unanticipated external noise.[1]

In this context, there are considerable advantages to tuning such a quantum transition in a system made of high temperature superconducting elements so that the intrinsic temperature scales and the scales of critical currents are large. (For this purpose, the fact that they are d-wave superconductors, or that the mechanism is unconventional are irrelevant details.) Such a study has been undertaken by Yang et al a paper that recently appeared on the arXiv. They have studied a large area (roughly $1 \mathrm{~mm} \times 1 \mathrm{~mm}$ ) $12 \mathrm{~nm}$ thick film of the cuprate high temperature YBCO patterned into an effective Josephson junction array by etching an array of order $10^{8}$ holes, each with approximately a $70 \mathrm{~nm}$ diameter with a mean center-to-center spacing of approximately 100nm. By ion bombardment, this system can be continuously tuned from a globally superconducting phase to a globally insulating one. Importantly, there is an intermediate regime in this evolution in which metallic behavior - 

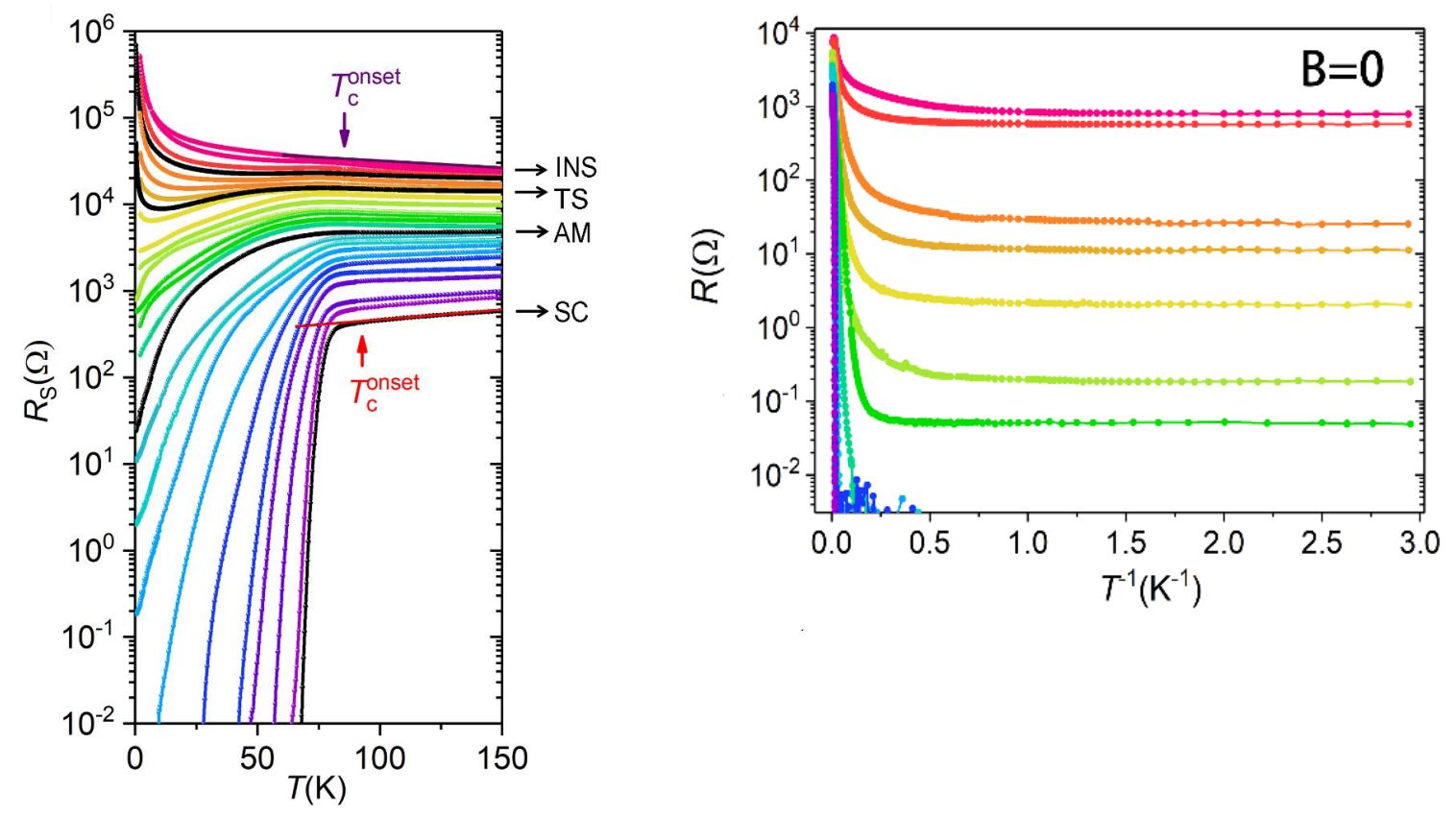

Figure 1: Two figures from the title article. 1) The left figure shows the log of the resistivity vs $T$ for various $b$. The black line labeled AM corresponds to $b=b_{c}$. The black line labeled TS corresponds to $b=b_{c 2}$. 2) The same curves in the SC and AM regimes $\left(b \leq b_{c 2}\right)$ plotted vs $1 / T$ to exhibit the low temperature saturation of the resistivity in the AM metal regime.

i.e. a resistivity that appears to approach a finite value as $T \rightarrow 0-$ is observed. Specifically, designating the degree to which the device has been subjected to ion bombardment as $b$, the device exhibits a finite $T$ superconducting transition for the $\mathrm{SC}$ regime, $0 \leq b<b_{c}$, exhibits a resistivity that is a decreasing function of decreasing $T$ that appears to approach a non-zero limit as $T \rightarrow 0$ in an anomalous metal (AM) regime, $b_{c}<b<b_{c 2}$, and finally enters a regime where the resistivity can even be non-monotonic, but where it appears to be diverging as $T \rightarrow 0$ in the insulating (I) regime, $b_{c 2}<b$.

In many ways, the behavior of this system is similar to that of a host of other 2D systems. However, there are a number of aspects of the results that are notable:

- 1) As advertised, the characteristic temperature scales are relatively large. At $b=0$, $T_{c} \approx 75 \mathrm{~K}$, and the resistivity curves in all but the most insulating regime begin to drop below their "normal state" value below an onset temperature of order $T_{c}(b=$ $0)$. Most importantly for present purposes, in the AM regime, the resistivity at low $T$ is sufficiently weakly $T$ dependent that it can be reasonably approximated as $T$ independent below temperatures as high as 5K. (This is a 10 to 100 times higher temperature scale than that which characterizes most of the other systems that have been studied in this context.[2])

- 2) Magneto-resistance oscillations with period corresponding to a superconducting flux quantum per hole have been observed in all three regimes. This directly establishes the existence of locally coherent charge $2 e$ collective modes (superconducting fluctu- 
ations) and their significance for charge transport. Needless to say, the temperature dependence of these oscillations is different in the three regimes: they increase steeply in magnitude with decreasing $T$ in the $\mathrm{SC}$ regime, decrease (albeit not so strongly) in the I regime, and are only weakly $T$ dependent at low $T$ in the AM regime.

- 3) The high temperature scale that characterizes the SC fluctuations in the AM regime can also be seen quite vividly in the $T$ dependence of the hall resistivity, $\rho_{x y}$, which plummets from its normal state value to become immeasurably small below approximately $50 \mathrm{~K}$. A similarly of high $T$ scale characterizes the positive magneto-resistance in the AM metal regime.

The fact that the temperature scales involved are so much larger than in other systems that have been studied in this context does not, of course, make the results proof against some of the possible extrinsic effects that could produce spurious results. These have been addressed directly as well as is currently possible, both by carefully studying the dependence of the measured resistivity on the current (to verify that the $I-V$ curves are linear) and by varying the degree to which external noise is excluded by performing experiments with and without an RC filter. By all these tests, the results seem robust.

The present results greatly enhance the strength of the evidence of the existence of a stable phase of matter in which there are substantial local superconducting correlations, but in which quantum fluctuations remain sufficiently strong that the system fails to condense into a globally phase coherent SC groundstate. If true, this conclusion is of fundamental importance for our understanding of quantum materials.

\section{References}

[1] I. Tamir, A. Benyamini, E.J. Telford, F. Gorniaczyk, A. Doron, T. Levinson, D. Wang, F. Gay, B. Sacepe, J. Hone, K.Watanabe, T. Taniguchi, C. R. Dean, A. N. Pasupathy,, and D. Shahar, Science Advances 5, 3826 (2019).

[2] For a recent review, see A. Kapitulnik, S. A. Kivelson, and B. I. Spivak, Rev. Mod. Phys. 91, 11002 (2019). 